\title{
2504. Free vibration of basalt fiber reinforced polymer (FRP) laminated variable thickness plates with intermediate elastic support using finite strip transition matrix (FSTM) method
}

\author{
Wael A. Altabey \\ International Institute for Urban Systems Engineering, Southeast University, Nanjing, 210096, China \\ Department of Mechanical Engineering, Faculty of Engineering, Alexandria University, \\ Alexandria, 21544, Egypt \\ E-mail:wael.altabey@gmail.com
}

Received 5 January 2017; received in revised form 29 January 2017; accepted 12 March 2017 DOI https://doi.org/10.21595/jve.2017.18154

\begin{abstract}
This paper presents a semi-analytical method to investigate the effect of intermediate elastic support on the natural frequencies of basalt fiber reinforced polymer (FRP) laminated, variable thickness plates based on the finite strip transition matrix (FSTM) method. The plate has a uniform thickness in $x$ direction and varying thickness $h(y)$ in $y$ direction. A singular value decomposition algorithm is employed at the intermediate support to eliminate the dependence of the solution of the first span on another span. By a new treatment of the intermediate line support, the dimension of the final matrix of the general solution will be the same as that of plates without intermediate support. Numerical results for different combinations of classical boundary conditions at the plate edges with different elastic restraint coefficients $\left(K_{T}\right)$ for intermediate elastic support are presented to obtain the first six frequency parameters. The illustrated results are in excellent agreement with solutions available in the literature, thus validating the accuracy and reliability of the proposed technique.
\end{abstract}

Keywords: free vibration, finite strip transition matrix, variable thickness plate, basalt FRP.

\section{Introduction}

Continuous plates and plates with intermediate stiffeners are very common in many engineering fields such as aerospace industries, civil engineering and marine engineering. Exact solutions of such plates are available only for some boundary conditions. For example, if two opposite sided are simply supported and the other sides may be any combinations of elastic, clamped and free, a Levy-type solution can be obtained for rigid stiffners [1].

In general, a numerical approach or an approximate method must be employed to find the natural frequencies and the mode shapes for different combinations of the boundary conditions. The vibration of plates with intermediate support attracts many researchers.

Xiang and Liew [1] presented an exact (Levy-type) solution for multi-span rectangular mindlin plates with two opposite edges simply supported. Abrate and Foster [2] used Rayleigh-Ritz method to investigate the free vibrations of rectangular composite plates with arbitrary number of intermediate line supports. Cheung and Zhou [3] used Rayleigh-Ritz method to study vibrations of symmetric laminated rectangular plates with intermediate supports. Liew and Wang [4] studied vibration of skew plates with internal line support using the pb-2 Rayleigh-Ritz method. Cheung and Zhou [5] used a set of static beam functions to analyze the vibration of orthotropic rectangular plates with intermediate elastic support. Xiang et al. [6] reported free vibration behavior of laminated seven composite plates based on the $n$th order shear deformation theory and this theory satisfies the zero transverse shear stress boundary conditions. Thai and Kim [7] examined the free vibration responses of laminated composite plates using two variables refined plate theory. Ovesy and Fazilati [8] employed the third order shear deformation theory for buckling and free vibration finite strip analysis of composite plates with cutout based on two different modeling approaches (semi-analytical and spline method). Dozio [9] presented accurate upper-bound solutions for free 
in-plane vibrations of single-layer and symmetrically laminated rectangular composite plates with an arbitrary combination of clamped and free boundary conditions. He used Rayleigh-Ritz method to calculate in-plane natural frequencies and modes shapes with a simple, stable and computationally efficient set of trigonometric functions. Asadi et al. [10] investigated the vibration analysis of axially moving functionally graded plates with internal line supports and temperaturedependent properties using harmonic differential quadrature method. They studied plate vibration which was subjected to static in-plane forces while out-of-plane loading was dynamic. Al-Tabey [11] presented the finite strip transition matrix technique (FSTM) and semi-analytical method to obtain the natural frequencies and mode shapes of symmetric angle-ply Graphite/Epoxy laminated composite variable thickness rectangular plate with classical boundary conditions (SSFF). Thinh et al. [12] examined the bending and vibration analysis of multi-folding laminate composite plate using finite element method based on the first order shear deformation theory (FSDT). They investigated the effect of folding angle on deflections, natural frequencies and transient displacement response for different boundary conditions of the plate. Ducceschi [13] studied the nonlinear vibrations of thin rectangular plates by developing of a numerical code able to simulate without restrictions. He described the large spectrum of dynamical features by the von Kármán equations. Yadav et al. [14] presented the free vibration analysis of stiffened isotropic plate by means of finite element method. They studied the effect of different boundary conditions, stiffeners location, thickness ratio, stiffener thickness to plate thickness and aspect ratio on the vibration analysis of stiffened isotropic plate, and calculated natural frequencies using Block-Lanczos algorithm. Küçükrendeci and Morgül [15] investigated the effects of elastic boundary conditions on the linear free vibrations. They found that frequency parameters increase when boron/epoxy used.

Semi-analytical methods are welcomed in the literature as an alternative to the exact solution. In this paper a semi-analytical method, the finite strips transition matrix (FSTM) method [16] has been employed to investigate the free vibration of basalt fiber reinforced polymer (FRP) laminated variable thickness rectangular plates with intermediate elastic support as shown in Fig. 1. A new treatment of the elastic intermediate boundary conditions using a singular values decomposition algorithm is introduced in this paper. Four different classical boundary conditions are considered in the analysis with different elastic restraint coefficients $\left(K_{T}\right)$ for intermediate elastic support to obtain the first six frequency parameters, some new data which can serve as the benchmark for further research are presented in this work.

\section{Theory and formulation}

\subsection{Governing equations}

The partial differential equation governing the vibration of symmetrically, angle-ply laminated, variable thickness, rectangular plates under the assumption of the classical deformation theory in terms of the plate deflection $w_{o}(x, y, t)$ is given by [17]:

$$
\begin{aligned}
& D_{11} \frac{\partial^{4} w_{o}}{\partial x^{4}}+4 D_{16} \frac{\partial^{4} w_{o}}{\partial x^{3} \partial y}+2\left(D_{12}+2 D_{66}\right) \frac{\partial^{4} w_{o}}{\partial x^{2} \partial y^{2}}+4 D_{26} \frac{\partial^{4} w_{o}}{\partial x \partial y^{3}}+D_{22} \frac{\partial^{4} w_{o}}{\partial y^{4}} \\
& \quad=-m_{o} \frac{h(y)}{h_{o}} \frac{\partial^{2} w_{o}}{\partial t^{2}} .
\end{aligned}
$$

Or in contraction form:

$$
\begin{aligned}
& D_{11} W_{x x x x}+4 D_{16} W_{x x x y}+2\left(D_{12}+2 D_{66}\right) W_{x x y y}+4 D_{26} W_{x y y y}+D_{22} W_{y y y y} \\
& \quad=-m_{o} \frac{h(y)}{h_{o}} W_{t t}
\end{aligned}
$$


where: $m_{o}=\rho h_{o}$, the flexural rigidities $D_{i j}$ of the plate are given by:

$D_{i j}=\frac{1}{3} \frac{h^{3}(y)}{h_{o}^{3}} \sum_{k=1}^{n}\left[\left(\bar{Q}_{i j}\right)\right]_{k}\left(h_{o k}^{3}-h_{o k-1}^{3}\right), \quad i, j=1,2,3$.

where $h_{o k}$ is the distance from the middle-plane of the plate according to $h_{o}$ to the bottom of the $h_{\text {oth }}$ layer as shown in Fig. 1. And $\overline{Q_{i j}^{k}}$ are the plane stress transformed reduced stiffness coefficients of the lamina in the laminate cartesian coordinate system. They are related to reduce stiffness coefficients of the lamina in the material axes of lamina $Q_{i j}^{k}$ by proper coordinate relationships they can be expressed in terms of the engineering notations as:

$Q_{i j}=\left[\begin{array}{lll}Q_{11} & Q_{12} & Q_{13} \\ Q_{12} & Q_{22} & Q_{23} \\ Q_{13} & Q_{23} & Q_{66}\end{array}\right]=\left[\begin{array}{ccc}\frac{E_{11}}{\left(1-v_{12} v_{21}\right)} & \frac{v_{21} E_{11}}{\left(1-v_{12} v_{21}\right)} & 0 \\ \frac{v_{21} E_{11}}{\left(1-v_{12} v_{21}\right)} & \frac{E_{22}}{\left(1-v_{21} v_{12}\right)} & 0 \\ 0 & 0 & G_{12}\end{array}\right]$,

where: $E_{11}, E_{22}$ are the longitudinal and transverse Young's moduli parallel and perpendicular to the fiber orientation, respectively and $G_{12}$ is the plane shear modulus of elasticity, $v_{12}$ and $v_{21}$ are the Poisson coefficients.

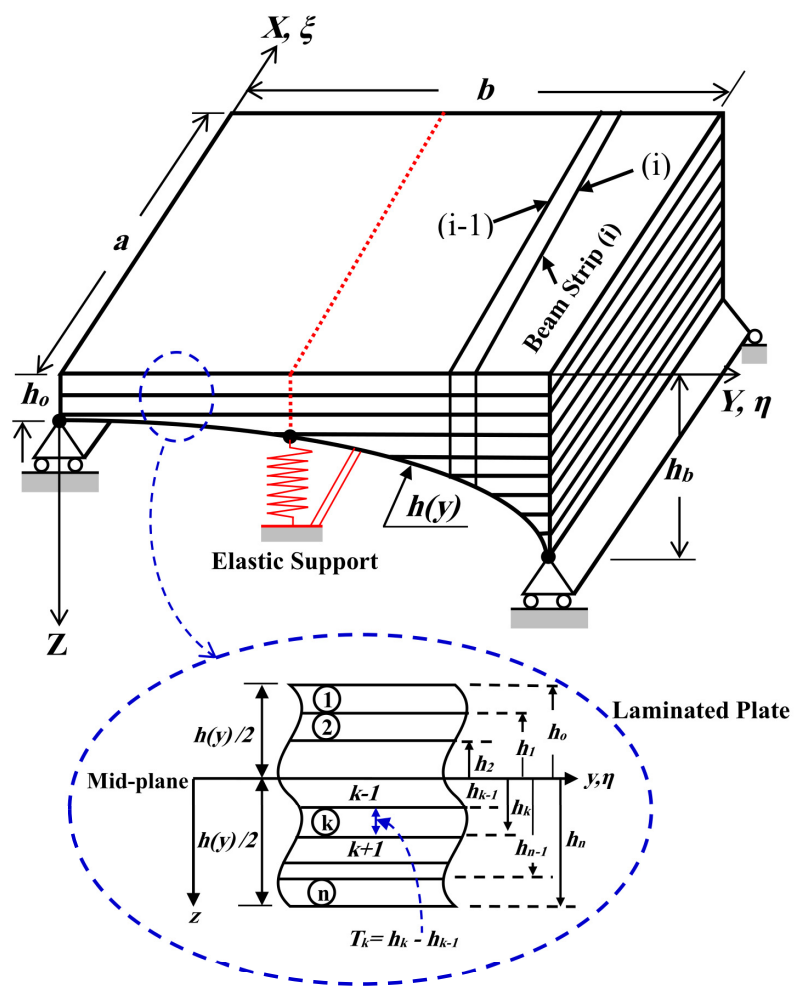

Fig. 1. The geometrical model of Basalt FRP laminated variable thickness rectangular plate with intermediate elastic support

The substitution of Eq. (3) into Eq. (2) and after some derivation steps [18], the governing Partial differential equation can be written in form: 


$$
\begin{aligned}
D_{11} & \frac{h^{3}(y)}{h_{o}^{3}} W_{x x x x}+\left(\frac{2\left(D_{12}+2 D_{66}\right)}{h_{o}^{3}}\right) \frac{\partial h^{3}(y)}{\partial y} W_{x x y}+\left(\frac{2\left(D_{12}+2 D_{66}\right)}{h_{o}^{3}}\right) h^{3}(y) W_{x x y y} \\
& +D_{16} \frac{h^{3}(y)}{h_{o}^{3}} W_{x x x y}+\left(\frac{4 D_{26}}{h_{o}^{3}} \frac{\partial^{2} h^{3}(y)}{\partial y^{2}}\right) W_{x y}+\frac{4 D_{26}}{h_{o}^{3}} h^{3}(y) W_{x y y y}+\frac{8 D_{26}}{h_{o}^{3}} \frac{\partial h^{3}(y)}{\partial y} W_{x y y} \\
& +\left(\frac{D_{22}}{h_{o}^{3}} \frac{\partial^{2} h^{3}(y)}{\partial y^{2}}\right) W_{y y}+\frac{D_{22}}{h_{o}^{3}} h^{3}(y) W_{y y y y}+\frac{2 D_{22}}{h_{o}^{3}} \frac{\partial h^{3}(y)}{\partial y} W_{y y y}=-m_{o} \frac{h(y)}{h_{o}} W_{t t} .
\end{aligned}
$$

The equation of motion Eq. (5) can be normalized using the non-Dimensional variables $\xi$ and $\eta$ as follows:

$$
\begin{aligned}
\psi_{1} & \frac{1}{a^{4}} W_{\xi \xi \xi \xi}+\frac{2 \psi_{2}}{h^{3}(\eta)} \frac{1}{a^{2} b} \frac{\partial h^{3}(\eta)}{\partial \eta} W_{\xi \xi \eta}+2 \psi_{2} \frac{1}{a^{2} b^{2}} W_{\xi \xi \eta \eta}+\psi_{3} \frac{1}{a^{3} b} W_{\xi \xi \xi \eta} \\
& +4 \psi_{4} \frac{1}{a b^{3}} W_{\xi \eta \eta \eta}+\frac{1}{a b} \frac{4 \psi_{4}}{h^{3}(\eta)} \frac{\partial^{2} h^{3}(\eta)}{\partial \eta^{2}} W_{\xi \eta}+\frac{8 \psi_{4}}{h^{3}(\eta)} \frac{1}{a b^{2}} \frac{\partial h^{3}(\eta)}{\partial \eta} W_{\xi \eta \eta} \\
& +\frac{1}{b^{2}} \frac{1}{h^{3}(\eta)} \frac{\partial^{2} h^{3}(\eta)}{\partial \eta^{2}} W_{\eta \eta}+\frac{1}{b^{4}} W_{\eta \eta \eta \eta}+\frac{2}{h^{3}(\eta)} \frac{1}{b^{3}} \frac{\partial h^{3}(\eta)}{\partial \eta} W_{\eta \eta \eta}=-\frac{m_{o}}{D_{22}} \frac{h_{o}^{2}}{h^{2}(\eta)} W_{t t}
\end{aligned}
$$

where $\beta=a / b$ is the aspect ratio, and:

$\xi=\frac{x}{a}, \quad \eta=\frac{y}{b}, \quad \psi_{1}=\frac{D_{11}}{D_{22}}, \quad \psi_{2}=\frac{\left(D_{12}+2 D_{66}\right)}{D_{22}}, \quad \psi_{3}=\frac{D_{16}}{D_{22}}, \quad \psi_{4}=\frac{D_{26}}{D_{22}}$.

\subsection{Boundary conditions}

In this paper, the boundary conditions along the $x$-direction and $y$-direction are considered by any combinations of the classical boundary conditions such as simply supported, clamped, or free. For the purpose of clarity, the symbol SFSC for example, means a plate having simply supported, free, simply supported and clamped edges at the boundaries, $x=0, y=b, x=a$, and $y=0$, respectively (start anticlockwise from the left edge of the plate). In the numerical computations, four different classical boundary conditions are considered in the analysis SSSS, CCCC, SSFF and CCFF as shown in Fig. 2.
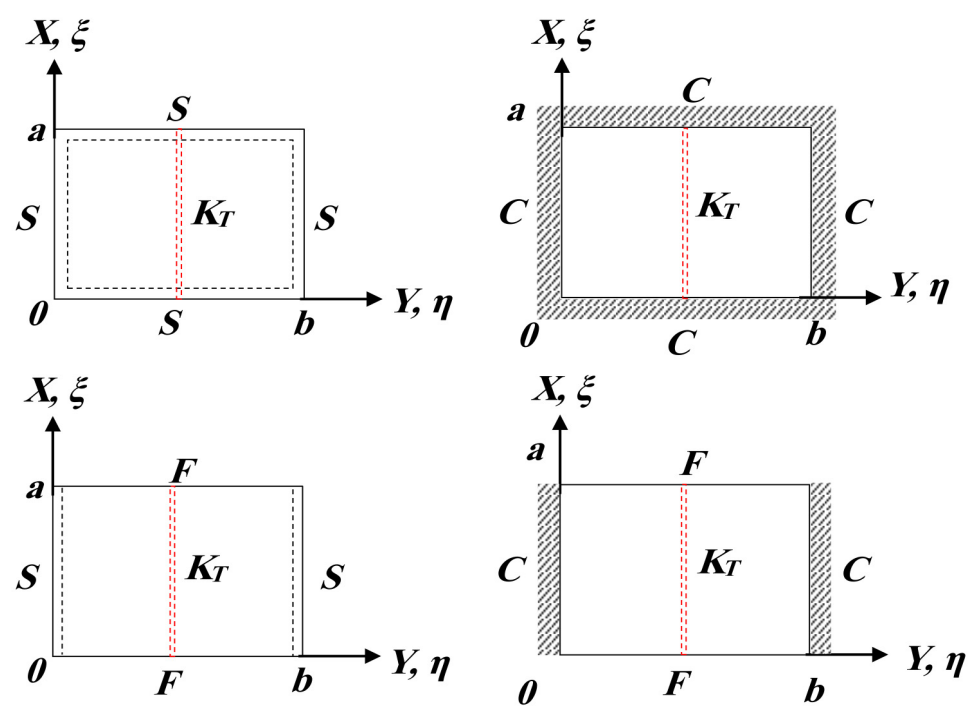

Fig. 2. Representation of different support condition for the analysis 
Simply supported edges:

$\left.w_{o}\right|_{\xi=0,1}=0,\left.\quad \frac{1}{a^{2}} \frac{\partial^{2} w_{o}}{\partial \xi^{2}}\right|_{\xi=0,1}=0,\left.\quad w_{o}\right|_{\eta=0,1}=0,\left.\quad \frac{1}{b^{2}} \frac{\partial^{2} w_{o}}{\partial \eta^{2}}\right|_{\eta=0,1}=0$.

Clamped supported edges:

$\left.w_{o}\right|_{\xi=0,1}=0,\left.\quad \frac{1}{a} \frac{\partial w_{o}}{\partial \xi}\right|_{\xi=0,1}=0,\left.\quad w_{o}\right|_{\eta=0,1}=0,\left.\quad \frac{1}{b} \frac{\partial w_{o}}{\partial \eta}\right|_{y=0,1}=0$.

Free edges:

$\left.\frac{1}{a^{3}} \frac{\partial^{3} w_{o}}{\partial \xi^{3}}\right|_{\xi=0,1}=0,\left.\quad \frac{1}{a^{2}} \frac{\partial^{2} w_{o}}{\partial \xi^{2}}\right|_{\xi=0,1}=0,\left.\quad \frac{1}{b^{3}} \frac{\partial^{3} w_{o}}{\partial \eta^{3}}\right|_{\eta=0,1}=0,\left.\quad \frac{1}{b^{2}} \frac{\partial^{2} w_{o}}{\partial \eta^{2}}\right|_{\eta=0,1}=0$.

\subsubsection{Intermediate elastic line support}

Since the treatment of the intermediate elastic line support conditions are the main objective of this paper we presented it in more details. At the intermediate elastic line support, $y=b / 2$, the displacement must vanish and the moment must be continuous, i.e.:

$$
\begin{aligned}
& K_{T} w_{o}=-2 \psi_{3} \frac{1}{a^{3}} \frac{\partial^{3} w_{o}}{\partial \xi^{3}}-\frac{1}{b^{3}} \frac{\partial^{3} w_{o}}{\partial \eta^{3}}-\psi_{5} \frac{1}{a^{2} b} \frac{\partial^{2} w_{o}}{\partial \xi^{2} \partial \eta}-4 \psi_{4} \frac{1}{a b^{2}} \frac{\partial^{3} w_{o}}{\partial \xi \partial \eta^{2}}, \\
& \left.\frac{1}{b} \frac{\partial w_{o}}{\partial \eta}\right|_{\eta=1^{-} / 2}=\left.\frac{1}{b} \frac{\partial w_{o}}{\partial \eta}\right|_{\eta=1^{+} / 2}, \\
& -2 \psi_{3} \frac{1}{a^{3}} \frac{\partial^{3} w_{o}}{\partial \xi^{3}}-\frac{1}{b^{3}} \frac{\partial^{3} w_{o}}{\partial \eta^{3}}-\psi_{5} \frac{1}{a^{2} b} \frac{\partial^{2} w_{o}}{\partial \xi^{2} \partial \eta}-\left.4 \psi_{4} \frac{1}{a b^{2}} \frac{\partial^{3} w_{o}}{\partial \xi \partial \eta^{2}}\right|_{\beta=1^{-} / 2} \\
& =-2 \psi_{3} \frac{1}{a^{3}} \frac{\partial^{3} w_{o}}{\partial \xi^{3}}-\frac{1}{b^{3}} \frac{\partial^{3} w_{o}}{\partial \eta^{3}}-\psi_{5} \frac{1}{a^{2} b} \frac{\partial^{2} w_{o}}{\partial \xi^{2} \partial \eta}-\left.4 \psi_{4} \frac{1}{a b^{2}} \frac{\partial^{3} w_{o}}{\partial \xi \partial \eta^{2}}\right|_{\beta=1^{+} / 2},
\end{aligned}
$$

where: $K_{T}$ is the elastic restraint coefficient given by: $K_{T}=T_{b / 2} b^{3} / D_{22}, T$ is translational stiffness per unit length, $\psi_{5}=\left(D_{12}+4 D_{66}\right) / D_{22}$.

\subsection{Finite strip transition matrix (FSTM) method}

The method is made when such a shape function is not conveniently obtained in case of discussing the plate problems by series. The plate may be divided into $N$ discrete longitudinal strips spanning between supports as shown in Fig. 3. Simple basic displacement interpolation functions may then be used to represent displacement field within and between individual strips.

For a plate striped in the $\xi$-direction as shown in Fig. 3, the shape function $W(\xi, \eta, t)$ may be assumed in the form:

$W(\xi, \eta, t)=\sum_{i=0}^{N} X_{i}(\xi) Y_{i}(\eta) e^{i \omega_{t}}$,

where: $Y_{i}(\eta)$ is unknown function to be determined and $X_{i}(\xi)$ is chosen a priori, the basic function in $\xi$-direction. The most commonly used is the Eigen function obtained from the solution of the differential equation of a beam vibration under the prescribed conditions of the stripe at $\xi=0$ and 
$\xi=1$. By substituting of Eq. (13) into Eq. (6), multiplying both sides by $X_{j}(x)$ and after some derivatives, we can find:

$$
\begin{aligned}
\sum_{i=0}^{N} & \sum_{j=0}^{M} \frac{\beta^{4}}{f_{3}(\eta)} Y_{i, \eta \eta \eta \eta}+2 \beta^{3} a \frac{f_{1}(\eta)}{f_{3}(\eta)} Y_{i, \eta \eta \eta} \\
& +\left(\frac{2 \psi_{2} \beta^{2}}{f_{3}(\eta)} \frac{c_{i j}}{a_{i j}}+8 \psi_{4} \beta^{2} a \frac{f_{1}(\eta)}{f_{3}(\eta)} \frac{b_{i j}}{a_{i j}}+\beta^{2} a^{2} \frac{f_{2}(\eta)}{f_{3}(\eta)}\right) Y_{i, \eta \eta} \\
& +\left(2 \psi_{2} \beta a \frac{f_{1}(\eta)}{f_{3}(\eta)} \frac{c_{i j}}{a_{i j}}+\frac{\psi_{3} \beta}{f_{3}(\eta)} \frac{d_{i j}}{a_{i j}}+4 \psi_{4} \beta a^{2} \frac{f_{2}(\eta)}{f_{3}(\eta)} \frac{b_{i j}}{a_{i j}}+\frac{4 \psi_{4} \beta^{3}}{f_{3}(\eta)} \frac{b_{i j}}{a_{i j}}\right) Y_{i, \eta} \\
& +\left(\frac{\psi_{1}}{f_{3}(\eta)} \frac{e_{i j}}{a_{i j}}-\Omega^{2}\right) Y_{i}=0,
\end{aligned}
$$

where:

$$
\begin{aligned}
& \Omega^{2}=\frac{m_{o} h(\eta) \omega^{2} a^{4}}{h_{o} D_{22}}, \quad f_{1}(\eta)=\frac{1}{h^{3}(\eta)} \frac{\partial h^{3}(\eta)}{\partial \eta}, \quad f_{2}(\eta)=\frac{1}{h^{3}(\eta)} \frac{\partial^{2} h^{3}(\eta)}{\partial \eta^{2}}, \\
& f_{3}(\eta)=\frac{h_{o}^{2}}{h^{2}(\eta)}, \quad a_{i j}=\int_{0}^{1} X_{i} X_{j} d \xi, \quad b_{i j}=\int_{0}^{1} X_{j} X_{i, \xi} d \xi, \\
& c_{i j}=\int_{0}^{1} X_{j} X_{i, \xi \xi} d \xi, \quad d_{i j}=\int_{0}^{1} X_{j} X_{i, \xi \xi \xi} d \xi, \quad e_{i j}=\int_{0}^{1} X_{j} X_{i, \xi \xi \xi \xi} d \xi .
\end{aligned}
$$

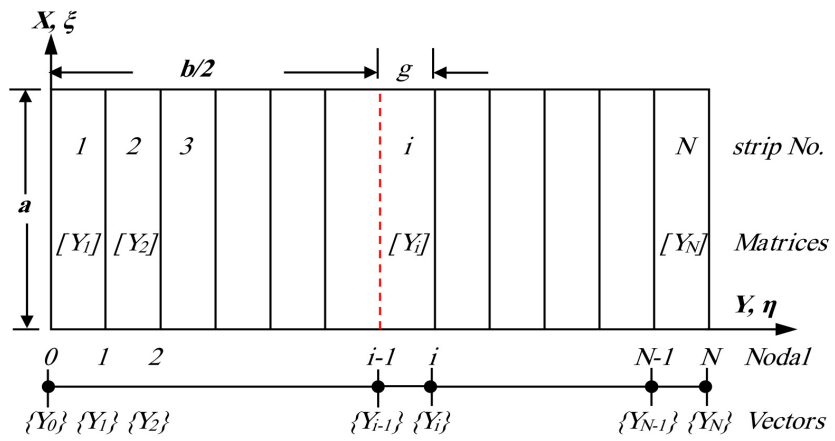

Fig. 3. Finite strip simulation on plate

From the beam Eigen function orthogonality, $a_{i j}=e_{i j}=0$ for $i \neq j$, this agree for all types of boundary conditions except for plates having free edges in the $\xi$-direction. The governing differential Eq. (14) can be written in form:

$\sum_{i=0}^{N} \sum_{j=0}^{M} E_{i j} Y_{i}^{\prime \prime \prime \prime}+\frac{\left(U_{1}\right)_{i j}}{\left(U_{0}\right)_{i j}} Y_{i}^{\prime \prime \prime}+\frac{\left(U_{2}\right)_{i j}}{\left(U_{0}\right)_{i j}} Y_{i}^{\prime \prime}+\frac{\left(U_{3}\right)_{i j}}{\left(U_{0}\right)_{i j}} Y_{i}^{\prime}+\frac{\left(U_{4}\right)_{i j}-\lambda^{2}}{\left(U_{0}\right)_{i j}} Y_{i}=0$,

where:

$\left(U_{0}\right)_{i j}=\beta^{4} t_{1}(\eta) E_{i j}, \quad\left(U_{1}\right)_{i j}=2 \beta^{3} a t_{2}(\eta) E_{i j}$,

$\left(U_{2}\right)_{i j}=\left(2 \psi_{2} \beta^{2} t_{1}(\eta) \frac{c_{i j}}{a_{i j}}+8 \psi_{4} \beta^{2} a t_{2}(\eta) \frac{b_{i j}}{a_{i j}}+\beta^{2} a^{2} t_{3}(\eta)\right)$, 


$$
\begin{aligned}
& \left(U_{3}\right)_{i j}=\left(2 \psi_{2} \beta a t_{2}(\eta) \frac{c_{i j}}{a_{i j}}+\psi_{3} \beta t_{1}(\eta) \frac{d_{i j}}{a_{i j}}+4 \psi_{4} \beta a^{2} t_{3}(\eta) \frac{b_{i j}}{a_{i j}}+4 \psi_{4} \beta^{3} t_{1}(\eta) \frac{b_{i j}}{a_{i j}}\right), \\
& \left(U_{4}\right)_{i j}=\psi_{1} t_{1}(\eta) \frac{e_{i j}}{a_{i j}}
\end{aligned}
$$

and $\left[E_{i j}\right]=i \times j$ unit matrix.

A system of coupled fourth order equations are obtained which can be reduced to a system of first order differential equation:

$$
\frac{d}{d \eta}\left\{Y_{k}\right\}_{i j}=\left[A_{i}\right]_{k}\left\{Y_{k}\right\}_{i j}
$$

where: $k=1,2,3, \ldots, N, i=1,2,3, \ldots, N, j=1,2,3, \ldots, M$, coefficients of the matrix $\left[A_{i}\right]_{k}$ in equation, in general, are functions of $\eta$ and the eigenvalue parameter $\Omega$. The vector $Y_{k}$ is given by:

$Y_{k}=\left(\begin{array}{llllll}\bar{Y}_{1} & \bar{Y}_{2} & \ldots & \bar{Y}_{i} & \ldots & \bar{Y}_{N}\end{array}\right)$

where:

$\bar{Y}_{i}=\left(\begin{array}{llll}Y_{i} & Y_{i}^{\prime} & Y_{i}^{\prime \prime} & Y_{i}^{\prime \prime \prime}\end{array}\right)$.

The relation under which the continuity conditions between the striped plates are satisfied may be expressed as:

$\left\{Y_{i}\right\}_{j}=\left[T_{i}\right]_{j}\left\{Y_{i-1}\right\}_{j}$

where: $\left[T_{i}\right]_{j}$ is called the transition matrix of the strip $i$ while $\left\{Y_{i}\right\}_{j}$ and $\left\{Y_{i-1}\right\}_{j}$ are the nodal vectors of the boundaries $i$ and $i-1$. The solution is found using $2 N$-number of initial vectors $\left\{Y_{0}\right\}$ at $\eta=0$. The transition matrix, Eq. (19) is applied across the stripped plate until just before the intermediate support at $y=b / 2, \eta=1 / 2$ is reached. Thus, $2 N$-number of solutions $S_{i}$ can be obtained. The true solutions $[S]$ can be written as a linear combination of these solutions as:

$[S]=\sum_{i=1}^{2 N} C_{i} S_{i}$,

where $C_{i}$ are arbitrary constants, these constants can be determined by satisfying $2 N$-number of boundary conditions at $\eta=1 / 2$ in Eqs. (10) and (12) of the intermediate elastic line support. And the matrix $[S]$ forms a standard eigenvalue problem. The natural frequencies of the system can be obtained from the conditions that the detainment of the $S$ must vanish. An iteration algorithm is implemented to compute the natural frequency of the system and hence the constants $C_{i}$, $i=1,2,3, \ldots, 2 N$.

\section{Results and discussion}

In this section, the finite strip transition matrix (FSTM) approach is employed to investigate the free vibration of symmetrically laminated, angle-ply, variable thickness rectangular plates with intermediate elastic support in one direction with different elastic restraint coefficient $\left(K_{T}\right)$. The basalt FRP laminate composite plate was manufactured using five symmetrically, angle-ply, laminates with the fiber orientations $\left[45^{\circ} /-45^{\circ} / 45^{\circ} /-45^{\circ} / 45^{\circ}\right]$ of basalt fiber and a polymer resin matrix. The corresponding elastic modulus values were $E_{1}=96.74 \mathrm{GPa}, E_{2}=E_{3}=22.55 \mathrm{GPa}$, and the Shear modulus values were $G_{1}=G_{3}=10.64 \mathrm{GPa}, G_{2}=8.73 \mathrm{GPa}$. Poisson coefficients 
were $v_{1}=v_{3}=0.3, v_{2}=0.6$ and the density was $2700 \mathrm{~kg} / \mathrm{m}^{3}$.

The frequency parameter $\Omega$ is evaluated in non-dimensional form, expressed as:

$\Omega=\sqrt{\frac{m_{o} h(\eta) \omega^{2} a^{4}}{h_{o} D_{22}}}$.

The plate with linear variable thickness, $h(y)$ is used (see Appendix) in non-dimensional form:

$h(\eta)=1+\Delta \eta$

where: $\Delta$ is the tapered ratio of plate given by $\Delta=\left(h_{b}-h_{o}\right) / h_{o},\left(h_{o}\right)$ is the thickness of the plate at $\eta=0$ and $\left(h_{b}\right)$ is the thickness of the plate at $\eta=1$.

\subsection{Convergence study and accuracy}

In this subsection, a convergence investigation is carried out for the proposed method, first six frequencies are calculated and compared with available results in literatures. Table 1 presents a convergence and comparison study for isotropic, square $(\beta=1.0)$, uniform thickness $(\Delta=0)$ plates with a mid-line support in each direction, the plate material has mechanical properties of $v_{1}=v_{2}=0.3, D_{11}=D_{22}=D=E h^{3} /\left[12\left(1-v^{2}\right)\right], D_{66}=(1-v) D / 2$. In this study the non-dimensional frequency parameter $\Omega$ become $\Omega=\left(\rho h \omega^{2} a^{4} / D\right)^{1 / 2}$. Two different classical boundary conditions are considered in the computational SSSS and CCCC. The computational results which are compared with values available from literatures [5, 19-21]. A very close agreement is observed.

Table 2 presented a convergence and comparison study for fully simply supported (SSSS) and fully clamped (CCCC) square $(\beta=1.0)$, uniform thickness $(\Delta=0)$ plates with elastic foundation support. The elastic coefficient is taken equal to 500, 1390.2 for SSSS and CCCC respectively. The plates are manufactured from E-glass/ epoxy material with the following properties are $v_{1}=v_{3}=0.23, D=E h^{3} /\left[12\left(1-v^{2}\right)\right], D_{66}=(1-v) D / 2$. In this study the non-dimensional frequency parameter $\Omega$ become $\Omega=\left(\rho h \omega^{2} a^{4} / \pi D\right)^{1 / 2}$ and foundation elastic restraint coefficient is given by $K_{T}=k_{f} a^{4} / D$. From Table 2 it can be observed that the computational results are in an excellent agreement with exact frequency parameters presented in References $[22,23]$ and stable and fast convergence can be achieved with only a few terms of series solution $(N=3$ to 7$)$. This validates the precision of the semi-analytical finite strip transition matrix (FSTM) technique.

Table 1. Convergence study of the first six frequency parameters of the isotropic square plates with a mid-line support in each direction

\begin{tabular}{|c|c|c|c|c|c|c|c|}
\hline & $N$ & $\Omega_{1}$ & $\Omega_{2}$ & $\Omega_{3}$ & $\Omega_{4}$ & $\Omega_{5}$ & $\Omega_{6}$ \\
\hline \multirow{4}{*}{ SSSS } & 1 & 78.866 & 94.506 & 94.506 & 108.125 & 197.311 & 197.311 \\
\cline { 2 - 8 } & 2 & 78.887 & 94.529 & 94.529 & 108.159 & 197.324 & 197.324 \\
\cline { 2 - 8 } & 4 & 78.910 & 94.546 & 94.546 & 108.184 & 197.350 & 197.350 \\
\cline { 2 - 8 } & 6 & 78.928 & 94.568 & 94.568 & 108.211 & 197.369 & 197.369 \\
\hline Ref [5] & & 78.957 & 94.590 & 94.590 & 108.240 & 197.392 & 197.392 \\
\hline Ref [19] & & 78.96 & 94.68 & 94.72 & 108.44 & 197.40 & 198.96 \\
\hline Ref [20] & & 78.958 & 94.826 & 94.826 & 108.41 & 197.50 & 197.50 \\
\hline Ref [21] & & 78.957 & 94.585 & 94.585 & 108.22 & 197.39 & 197.33 \\
\hline & 2 & 108.222 & 127.346 & 127.346 & 144.026 & 242.386 & 242.386 \\
\cline { 2 - 8 } CCCC & 4 & 108.243 & 127.365 & 127.365 & 144.048 & 242.758 & 242.758 \\
\cline { 2 - 8 } & 5 & 108.259 & 127.382 & 127.382 & 144.071 & 242.773 & 242.773 \\
\cline { 2 - 8 } & 7 & 108.282 & 127.398 & 127.398 & 144.099 & 242.801 & 242.801 \\
\hline Ref [5] & & 108.299 & 127.417 & 127.417 & 144.109 & 242.818 & 243.778 \\
\hline
\end{tabular}


Table 2. Convergence study of the first four frequency parameters of the isotropic square plates with elastic foundation

\begin{tabular}{|c|c|c|c|c|c|c|}
\hline & $N$ & $K_{T}$ & $\Omega_{1}$ & $\Omega_{2}$ & $\Omega_{3}$ & $\Omega_{4}$ \\
\hline \multirow{4}{*}{ SSSS } & 2 & 500 & 3.0210 & 5.4828 & 5.4828 & 8.3017 \\
\cline { 2 - 7 } & 3 & 500 & 3.0211 & 5.4836 & 5.4836 & 8.3019 \\
\cline { 2 - 7 } & 4 & 500 & 3.0212 & 5.4842 & 5.4842 & 8.3023 \\
\cline { 2 - 7 } & 7 & 500 & 3.0213 & 5.4847 & 5.4847 & 8.3029 \\
\hline Ref [22] & & 500 & 3.0214 & 5.4850 & 5.4850 & 8.3035 \\
\hline Ref [23] & & 500 & 3.0216 & 5.4846 & 5.4846 & 8.3051 \\
\hline \multirow{5}{*}{ CCCC } & 2 & 1390.2 & 5.2515 & 8.3785 & 8.3785 & 11.506 \\
\cline { 2 - 7 } & 3 & 1390.2 & 5.2538 & 8.3811 & 8.3811 & 11.528 \\
\cline { 2 - 7 } & 6 & 1390.2 & 5.2554 & 8.3843 & 8.3843 & 11.553 \\
\cline { 2 - 7 } & 7 & 1390.2 & 5.2573 & 8.3879 & 8.3879 & 11.568 \\
\hline Ref [22] & & 1390.2 & 5.2588 & 8.4322 & 8.4322 & 11.674 \\
\hline Ref [23] & & 1390.2 & 5.2438 & 8.3129 & 8.3129 & 11.546 \\
\hline
\end{tabular}

\subsection{Laminated variable thickness plate with intermediate elastic line support}

The results from the numerical computations using FSTM approach will be discussed here. Table 3 presents the first six frequencies of a symmetrically, angle-ply, laminated, variable thickness rectangular plate with intermediate elastic line support in one direction as shown in Fig. 1. The aspect ratio of the plate is $\beta=0.5$ and tapered ratio of the plate thickness is $\Delta=0.5$. Four type of classical boundary conditions (SSSS, CCCC, SSFF and CCFF) as shown in Fig. 2 and different elastic restraint coefficients $K_{T}$ of intermediate elastic line support are considered in the computations to study the effect of intermediate elastic support on the natural frequencies of basalt (FRP) laminated variable thickness rectangular plate. The locations of the intermediate elastic line support is at mid-line of the plate.

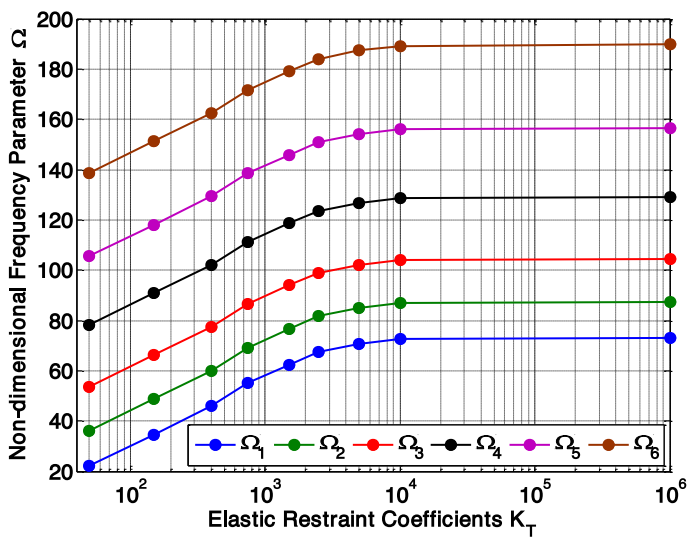

Fig. 4. Variation of non-dimensional frequencies parameter $(\Omega)$ with elastic restraint coefficient $\left(K_{T}\right)$

The effect of intermediate elastic support on the non-dimensional frequencies of laminated variable thickness rectangular plate is computed and plotted in Figs. 4 and 5. From this figures, it is observed that the first six frequencies increase with the increasing of the value of elastic restraint coefficient $\left(K_{T}\right)$ as shown in Fig. 4. Fig. 5 shows the vibration behaviour of the variable thickness rectangular plate under varying elastic restraint coefficient $\left(K_{T}\right)$. As shown in the Fig. 5, the increasing values of frequencies with small elastic restraint coefficient $\left(K_{T}\right)$ are higher than the increasing values of frequencies with highest one, and the frequencies at high values of elastic restraint coefficient are almost constant.

After the value of $K_{T}$ increases from 50 onwards, the non-dimensional frequencies parameter are fast raised till value of $K_{T}$ reached $10^{4}$ and after this value there is almost negligible change in 
value of Non-dimensional frequencies parameter.

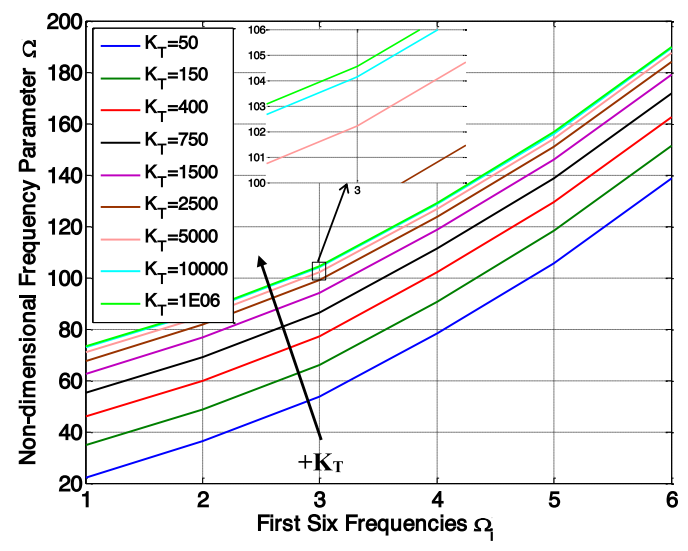

Fig. 5. Variation of non-dimensional frequencies parameter $(\Omega)$ with different mode number and elastic restraint coefficient $\left(K_{T}\right)$

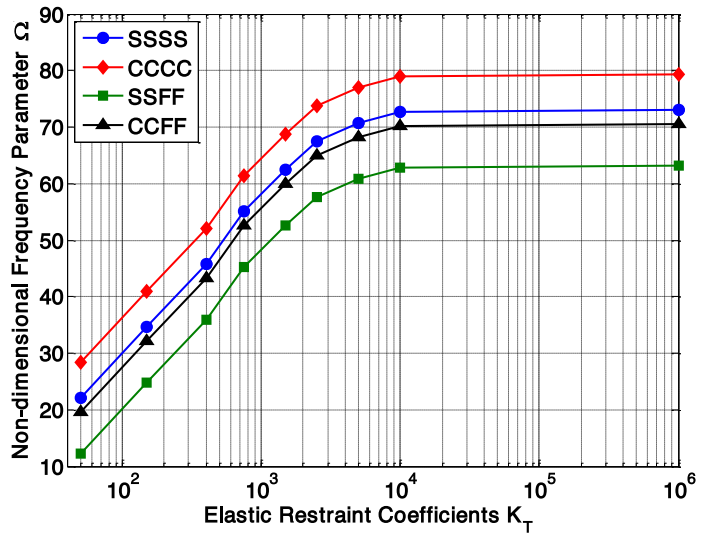

Fig. 6. Variation of non-dimensional frequencies parameter $(\Omega)$ with elastic restraint coefficient $\left(K_{T}\right)$ and boundary conditions

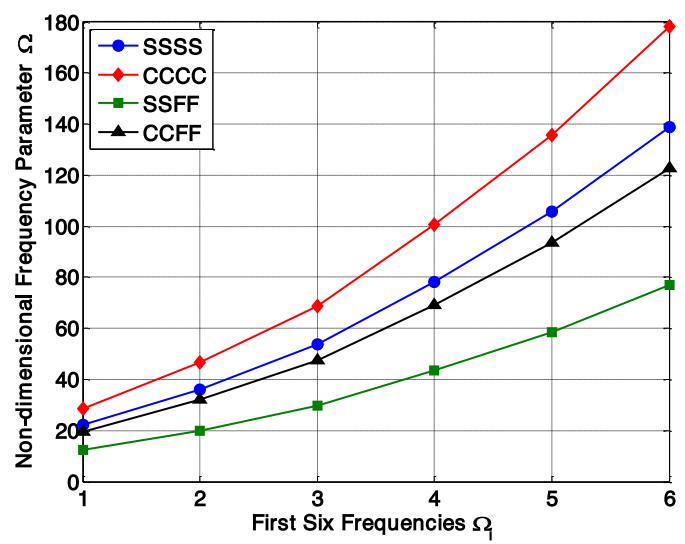

Fig. 7. Variation of non-dimensional frequencies parameter $(\Omega)$ with different mode number and boundary conditions

Influence of four different support conditions (SSSS, CCCC, SSFF and CCFF) on the vibration behavior of a symmetrically, angle-ply, laminated, variable thickness rectangular plate is 
computed and plotted in Figs. 6 and 7, From this figures, it can be seen that the frequencies are showing higher and lower value at fully clamped (CCCC) and semi-simply supported (SSFF) condition, respectively. The other two boundary conditions (SSSS and CCFF) are showing an intermediate value. As shown in the Fig. 6, the non-dimensional frequencies increase with the increase of the elastic restraint coefficient $\left(K_{T}\right)$ for all kind of support conditions (SSSS, CCCC, SSFF and CCFF).

Table 3. The first six frequencies of symmetrically, angle-ply, laminated, variable thickness rectangular plate with intermediate elastic line support for different elastic restraint coefficients, $(\Delta=0.5),(\beta=0.5)$

\begin{tabular}{|c|c|c|c|c|c|c|c|}
\hline & $K_{T}$ & $\Omega_{1}$ & $\Omega_{2}$ & $\Omega_{3}$ & $\Omega_{4}$ & $\Omega_{5}$ & $\Omega_{6}$ \\
\hline \multirow{9}{*}{ SSSS } & 50 & 22.1450 & 36.2210 & 53.5870 & 78.2360 & 105.5870 & 138.6970 \\
\hline & 150 & 34.6580 & 48.7340 & 66.1000 & 90.7490 & 118.1000 & 151.2100 \\
\hline & 400 & 45.8453 & 59.9213 & 77.2873 & 101.9363 & 129.2873 & 162.3973 \\
\hline & 750 & 55.0692 & 69.1452 & 86.5112 & 111.1602 & 138.5112 & 171.6212 \\
\hline & 1500 & 62.4709 & 76.5469 & 93.9129 & 118.5619 & 145.9129 & 179.0229 \\
\hline & 2500 & 67.5270 & 81.6030 & 98.9690 & 123.6180 & 150.9690 & 184.0790 \\
\hline & 5000 & 70.7832 & 84.8592 & 102.2252 & 126.8742 & 154.2252 & 187.3352 \\
\hline & 10000 & 72.7065 & 86.7825 & 104.1485 & 128.7975 & 156.1485 & 189.2585 \\
\hline & $1 \mathrm{E}+06$ & 73.1195 & 87.1955 & 104.5615 & 129.2105 & 156.5615 & 189.6715 \\
\hline \multirow{9}{*}{$\mathrm{CCCC}$} & 50 & 28.4310 & 46.5025 & 68.7979 & 100.4437 & 135.5584 & 178.0668 \\
\hline & 150 & 40.9440 & 59.0155 & 81.3109 & 112.9567 & 148.0714 & 190.5798 \\
\hline & 400 & 52.1313 & 70.2028 & 92.4983 & 124.1440 & 159.2587 & 201.7672 \\
\hline & 750 & 61.3551 & 79.4267 & 101.7221 & 133.3678 & 168.4826 & 210.9910 \\
\hline & 1500 & 68.7569 & 86.8284 & 109.1239 & 140.7696 & 175.8843 & 218.3928 \\
\hline & 2500 & 73.8130 & 91.8845 & 114.1800 & 145.8257 & 180.9404 & 223.4489 \\
\hline & 5000 & 77.0692 & 95.1407 & 117.4361 & 149.0819 & 184.1966 & 226.7050 \\
\hline & 10000 & 78.9925 & 97.0640 & 119.3594 & 151.0052 & 186.1199 & 228.6283 \\
\hline & $1 \mathrm{E}+06$ & 79.4055 & 97.4770 & 119.7724 & 151.4182 & 186.5329 & 229.0413 \\
\hline \multirow{9}{*}{ SSFF } & 50 & 12.2750 & 20.0773 & 29.7033 & 43.3663 & 58.5270 & 76.8799 \\
\hline & 150 & 24.7880 & 32.5903 & 42.2163 & 55.8793 & 71.0400 & 89.3929 \\
\hline & 400 & 35.9753 & 43.7777 & 53.4036 & 67.0666 & 82.2273 & 100.5802 \\
\hline & 750 & 45.1992 & 53.0015 & 62.6275 & 76.2905 & 91.4511 & 109.8041 \\
\hline & 1500 & 52.6009 & 60.4033 & 70.0293 & 83.6922 & 98.8529 & 117.2058 \\
\hline & 2500 & 57.6570 & 65.4594 & 75.0853 & 88.7483 & 103.9090 & 122.2619 \\
\hline & 5000 & 60.9132 & 68.7155 & 78.3415 & 92.0045 & 107.1652 & 125.5181 \\
\hline & 10000 & 62.8365 & 70.6388 & 80.2648 & 93.9278 & 109.0885 & 127.4414 \\
\hline & $1 \mathrm{E}+06$ & 63.2495 & 71.0518 & 80.6778 & 94.3408 & 109.5015 & 127.8544 \\
\hline \multirow{9}{*}{$\mathrm{CCFF}$} & 50 & 19.5928 & 32.0466 & 47.4112 & 69.2194 & 93.4182 & 122.7123 \\
\hline & 150 & 32.1058 & 44.5596 & 59.9242 & 81.7324 & 105.9312 & 135.2253 \\
\hline & 400 & 43.2931 & 55.7469 & 71.1115 & 92.9197 & 117.1185 & 146.4127 \\
\hline & 750 & 52.5170 & 64.9707 & 80.3353 & 102.1436 & 126.3424 & 155.6365 \\
\hline & 1500 & 59.9187 & 72.3725 & 87.7371 & 109.5453 & 133.7442 & 163.0383 \\
\hline & 2500 & 64.9748 & 77.4286 & 92.7932 & 114.6014 & 138.8002 & 168.0944 \\
\hline & 5000 & 68.2310 & 80.6848 & 96.0494 & 117.8576 & 142.0564 & 171.3505 \\
\hline & 10000 & 70.1543 & 82.6081 & 97.9727 & 119.7809 & 143.9797 & 173.2738 \\
\hline & $1 \mathrm{E}+06$ & 70.5673 & 83.0211 & 98.3857 & 120.1939 & 144.3927 & 173.6868 \\
\hline
\end{tabular}

\section{Conclusions}

The work reported in this paper employs an efficient semi-analytical method for analysing the free vibration of thin basalt fiber reinforced polymer (FRP) laminated variable thickness rectangular plates with intermediate elastic support. A singular value decomposition algorithm has been employed to treat the intermediate support and reduce the dependence of the solutions at the intermediate elastic support. It is observed that the first six frequencies increase with increasing 
values of elastic restraint coefficient $\left(K_{T}\right)$ of intermediate elastic support, and the rate of increasing is different. It was found that the increasing rates of frequencies with a small elastic restraint coefficient $\left(K_{T}\right)$ are higher than the increasing rates of frequencies with highest one, and the frequencies at high values of elastic restraint coefficient are almost constant. On other hand, it observed that the frequencies values were influenced with change of the plate edges support between four different support conditions, for all first six frequencies are showing higher and lower value at fully clamped (CCCC) and semi-simply supported (SSFF) condition, respectively, the other two boundary conditions (SSSS and CCFF) are showing an intermediate value. Accuracy and convergence of solution was examined by comparing the numerical results obtained by the present method with those previously published. The results are in excellent agreement with results from the literature.

\section{References}

[1] Xiang Y., Wei G. W. Exact solutions for multi-span rectangular Mindlin plates. Journal of Vibration and Acoustics, Vol. 124, 2002, p. 545-551.

[2] Abrate S., Foster E. Vibration of composite plates with intermediate line supports. Journal of Sound and Vibration, Vol. 179, 1995, p. 793-815.

[3] Cheung Y. K., Zhou D. Vibration of rectangular plates with elastic intermediate line-supports and edges constraints. Journal of Thin-walled Structures, Vol. 37, 2000, p. 305-331.

[4] Liew K. M., Wang C. M. Vibration studies on skew plates: treatment of internal line supports. Journal of Computer and Structures, Vol. 49, Issue 6, 1993, p. 941-951.

[5] Cheung Y. K., Zhou D. Vibration analysis of Symmetrically Laminated rectangular plates with intermediate line supports. Journal of Computer and Structures, Vol. 79, 2001, p. 33-41.

[6] Xiang S., Jiang S., Bi Z., Jin Y., Yang M. A nth-order meshless generalization of Reddy's third order shear deformation theory for the free vibration on laminated composite plates. Journal of Composite Structures, Vol. 93, 2011, p. 299-307.

[7] Thai H., Kim S. Free vibration of laminated composite plates using two variable refined plate theory. International Journal of Mechanical Sciences, Vol. 52, 2010, p. 626-633.

[8] Ovesy H. R., Fazilati J. Buckling and free vibration finite strip analysis of composite plates with cutout based on two different modeling approaches. Journal of Composite Structures, Vol. 94, 2012, p. $1250-1258$.

[9] Dozio L. In-plane free vibrations of single-layer and symmetrically laminated rectangular composite plates. Journal of Composite Structures, Vol. 93, 2011, p. 1787-1800.

[10] Asadi H., Aghdam M. M., Shakeri M. Vibration analysis of axially moving line supported functionally graded plates with temperature-dependent properties. Journal of Mechanical Engineering Science, Vol. 228, Issue 6, 2014, p. 953-969.

[11] Al-Tabey W. A. Vibration Analysis of Laminated Composite Variable Thickness Plate Using Finite Strip Transition Matrix Technique and MATLAB Verifications, book MATLAB Applications for the Practical Engineer. Technology and Medicine Open Access Book Publisher, 2014, p. 283-620.

[12] Thinh T. I., Binh B. V., Tu T. M. Bending and vibration analysis of multi-folding laminate composite plate using finite element method. Vietnam Journal of Mechanics, Vol. 34, Issue 3, 2012, p. 185-202.

[13] Ducceschi M. Nonlinear Vibrations of Thin Rectangular Plates: A Numerical Investigation with Application to Wave Turbulence and Sound Synthesis. Ph.D. Thesis, ENSTA-Paris Tech, 2014.

[14] Yadav D. P. S., Sharma A. K., Shivhare V. Free vibration analysis of isotropic plate with stiffeners using finite element method. Journal of Engineering Solid Mechanics, Vol. 3, 2015, p. 67-176.

[15] Küçükrendeci I., Morgül Ö. K. The effects of elastic boundary conditions on the linear free vibrations. Journal of Scientific Research and Essays, Vol. 6, Issue 19, 2001, p. 3949-3958.

[16] Bert W., Malik M. Free vibration analysis of tapered rectangular plates by differential quadrature method: a semi-analysis approach. Journal of Sound and Vibration, Vol. 190, 1996, p. 41-63.

[17] Chakraverty S. Vibration of Plates. CRC Press, Taylor and Francis Group, 2009.

[18] Reddy J. N. Mechanics of Laminated Composite Plates and Shells Theory and Analysis. 2nd Ed., CRC Press, Boca Raton London New York Washington, D.C., 2006.

[19] Wu C. I., Cheung Y. K. Frequency analysis of rectangular plates continuous in one or two directions. Journal of Earthquake Engineering Structure Dynamics, Vol. 3, 1974, p. 3-14. 
[20] Kim C. S., Dickinson S. M. The flexural vibration of line supported rectangular plate systems. Journal of Sound Vibration, Vol. 114, 1987, p. 29-42.

[21] Leissa A. W. The free vibration of rectangular plates. Journal of Sound and Vibration, Vol. 31, 1973, p. 257-93.

[22] Zhou D., Cheung Y. K., Lo S. H., Au F. T. K. Three dimensional vibration analysis of rectangular thick plates on Pasternak foundation. International Journal of Numerical Methods in Engineering, Vol. 59, 2004, p. 1313-1334.

[23] Ferreira J. M., Roque C. M. C., Neves A. M. A., Jorge R. M. N., Soares, C. M. M. Analysis of plates on Pasternak foundations by radial basis functions. Journal of Computational Mechanics, Vol. 46, 2010, p. 791-803.

\section{Appendix}

In this appendix the plate thickness function $h(y)$ in $y$-direction will be investigated of the as shown in the Fig. 8 is given.

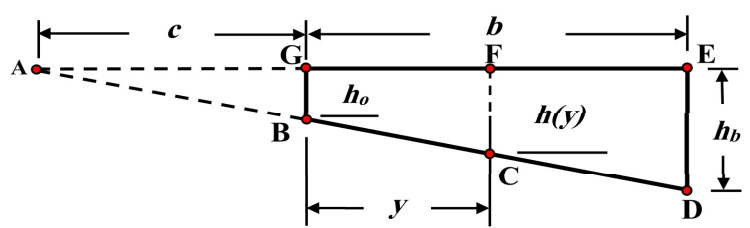

Fig. 8. The plate thickness $h(y)$ in $y$-direction

By similarity between the triangles $(\mathrm{ABG})$ and $(\mathrm{ACF})$ :

$h(y)=h_{o}\left(1+\frac{y}{c}\right)$.

By similarity between the triangles (ABG) and (ADE):

$\frac{h_{o}}{c}=\frac{h_{b}}{c+b}$.

From Eqs. (23) and (24) the plate thickness function is:

$h(y)=h_{o}+\frac{\left(h_{b}-h_{o}\right)}{b} y$,

where: $h(y)=h_{o}$ at $y=0, h(y)=h_{b}$ at $y=b, h(y)=h_{o}+\frac{\left(h_{b}-h_{o}\right)}{b} y$ at $y=y$, and $h(y)=h$ at $h_{o}=h_{b}$.

Using the assumed solution, Eq. (13), the thickness of the plate $h(y)$ can be given by the following equation:

$h(\eta)=h_{o}+\left(h_{b}-h_{o}\right) \eta$.

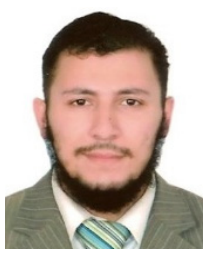

Wael A. Altabey is an Assistant Professor, since 2015 at Mechanical Engineering Department, Faculty of Engineering, Alexandria University, Alexandria, Egypt. He is currently a postdoctoral research fellow in International Institute for Urban Systems Engineering, Southeast University, Nanjing, China. He received his Ph.D., 2015 in Fatigue of Composite Structures and his M.Sc., 2009, in Dynamic Systems and his B.Sc., 2004, in Mechanical Engineering, from Mechanical Engineering Department, Faculty of Engineering, Alexandria University. 